\title{
THE EFFECT OF OXIDATIVE STRESS ON MITOCHONDRIAL ULTRASTRUCTURE AND IMMUNOEXPRESSION OF ERK-1 AND HIF-1 ALPHA IN ORAL EPITHELIAL DYSPLASIA INDUCED IN EXPERIMENTAL ANIMALS
}

\author{
Nermine Raouf Amin*, Safa Fathy Abdel Ghany ${ }^{* *}$ and Radwa Hamed Hegazi***
}

\begin{abstract}
Background: Oxidative stress and accumulation of reactive oxygen species may play a role in the pathogenesis of cancer. They were reported in precancerous and cancerous cells.

Methods: An animal model of carcinogenesis was used to detect the effect of oxidative stress on mitochondrial ultrastructure by transmission electron microscope and the levels of extracellular signal- regulated kinase-1(ERK-1) and hypoxia inducible factor-1 alpha (HIF-1 $\alpha$ ) by immunohistochemistry in mild and severe oral epithelial dysplasia (OED) induced in tongues of experimental mice.
\end{abstract}

Results: We identified degeneration and enlargement of mitochondria in association with upregulation of both ERK-1 and HIF-1 $\alpha$ from normal control to mild OED and reached their highest values in severe OED.

Conclusions: Degeneration and enlargement of mitochondria in association with up-regulation of both ERK-1 and HIF-1 $\alpha$ can be indicative features of dysplastic changes and may act as early markers for malignancy.

KEYWORDS: Oral epithelial dysplasia; oxidative stress; extracellular signal-regulated kinase -1 (ERK-1); hypoxia inducible factor-1 alpha (HIF-1 $\alpha$ ).

Lecturer of Oral Pathology, Faculty of Oral and Dental Medicine, Cairo University

** Assistant Professor of Oral Pathology, Faculty of Oral and Dental Medicine, Cairo University

***Assistant Professor of Oral Biology, Faculty of Oral and Dental Medicine, Cairo University 


\section{INTRODUCTION}

Oxidative stress implies a cellular state where reactive oxygen species (ROS) production exceeds the cell's ability to metabolize them resulting in excessive accumulation of ROS which overwhelms cellular defenses (Ziech et al., 2011). Elevated levels of ROS were reported in precancerous and cancerous cells; which is attributed to active metabolism and dysregulation of several cellular events in these dysplastic cells, thus being finally under constant oxidative stresses (Hileman et al., 2004 and Vairaktaris et al., 2008).

This constant oxidative stress can activate a variety of transcription factors leading to the expression of many growth factors, inflammatory cytokines, cell cycle regulatory molecules and anti-inflammatory molecules (Reuter et al., 2010). Moreover, it may cause DNA, protein and/or lipid damage, leading to changes in chromosome stability, genetic mutation and/or modulation of cell growth which may play a role in the pathogenesis of cancer (Pavithra, 2014).

The mitochondrion, the powerhouse of the cell, is one of the main sources where production of ROS takes place. That's why it is a primary target for oxidative damage, resulting from accumulation of ROS in its matrix. Thus, it can function as an endogenous oxygen sensor (Wang et al., 2000 and $\mathrm{Yu}, 2011)$. Recent studies showed that increased level of mitochondrial ROS promotes overexpression of extracellular signal-regulated kinase-1 (ERK-1) in many cancers (Thannickal and Fanburg, 2000)

ERK-1 is a member of mitogen-activated protein (MAP) kinase pathway; it is activated in response to various mitogenic factors, differentiation stimuli and cytokines. The ERK-1/2 MAP kinase pathway has emerged as a central regulator of cell proliferation by controlling both cell growth and cell cycle progression, in addition to inactivation of antipro- liferative genes (Meloche and Pouysségur, 2007). ERK-1 activation is able to directly phosphorlylate the carboxy-terminal domain of hypoxia inducible factor-1 alpha (HIF-1 $\alpha$ ) (Lin et al., 2008) and stimulate its transcriptional activity (Dimova et al., 2009).

HIF- $1 \alpha$ is considered as the master transcriptional regulator of cellular response to hypoxia. It accumulates under low-oxygen conditions and drives the production of stress-adaptive proteins. This response is essential for maintenance of normal oxidative physiology; however its overexpression in cancer cells has promoted tumor survival. The dysregulation and overexpression of HIF- $1 \alpha$ have been heavily implicated in cancer biology (Tilakaratne \& Nissanka-Jayasuriya, 2011).

Despite the interest in exploring the effect of oxidative stress and its underlying molecularchanges in oral epithelial dysplasia (OED), this field is not adequately addressed. Accordingly, our goal was to study the effect of oxidative stress on mitochondrial ultrastructure in relation to ERK-1 and HIF- $1 \alpha$ immunoexpression in OED induced in tongues of experimental mice in an attempt to understand their contribution to the development and progression of OED and their potential role as early markers for detection of malignant transformation.

\section{MATERIAL AND METHODS}

\section{Experimental animals}

The current study was carried out on 60 mice weighing $250 \pm 10 \mathrm{gm}$. They were maintained in the animal house as an inbred colony, obtained from Faculty of Medicine, Cairo University, Egypt. Their ages ranged from 6-8 weeks; they were housed in stainless-steel cages, 5 animals per cage. They were allowed to grow under the same conditions of temperature $\left(20^{\circ}-26^{\circ} \mathrm{C}\right)$ and humidity $(40-70 \%)$ under a 12/12h light/dark cycle. 


\section{Experimental design}

The selected experimental mice were randomly divided into two main groups (30 mice each): Control group (C group) and Experimental group (E group). In the E group, squamous cell carcinogenesis was induced in the tongues of the experimental mice. On the other hand, the $\mathrm{C}$ group was not exposed to any induction of carcinogenesis. Fifteen mice from each group were sacrificed ethically at 6-, and 9 -week intervals from the beginning of induction of carcinogenesis.

\section{Induction of squamous cell carcinogenesis}

DMBA (7,12-dimethylbenz[a]anthracene) and formaldehyde were used to induce carcinogenesis in the experimental animals. These carcinogens were purchased from Sigma-Aldrich Chemical Pte Ltd, Cairo, Egypt. The dorsal and ventral surfaces of tongues of the animals were painted with $0.5 \%$ DMBA in acetone 3 days/week. Two weeks later, $10 \%$ formaldehyde/water was used in combination with DMBA. Prior to carcinogens application, mice were anesthetized through intramuscular injection of Ketalar solution with a dose of $0.3 \mathrm{ml} / 100 \mathrm{gm}$.

\section{Experimental animal sacrifice}

The experimental animals were sacrificed by an overdose of Ketalar anesthetic solution ( $1 \mathrm{ml} / 100 \mathrm{gm})$ according to the research animal guidelines of Euthanasia University of Minnesota (2009). The current experimental protocol was approved by the Department of Animal Care, Cairo University that adhered to the European Communities Council guiding principles for the care and use of laboratory animals. Besides, an ethical approval was given by the ethics committee in the Faculty of Oral and Dental Medicine, Cairo University.

\section{Histological assessment of tissue changes}

The tongue specimens taken from sacrificed animals at different week intervals were prepared to obtain paraffin-embedded tissue sections. Small parts of one cubic mm were cut from each specimen (areas of interest) to be used for examination by the transmission electron microscopy. The paraffin-embedded tissue sections were stained with hematoxylin and eosin and studied under ordinary light microscope to monitor the production of epithelial dysplasia and invasive carcinoma. The diagnosis and grading of epithelial dysplasia was based on a combination of architectural and cytological changes as adopted by the WHO criteria (Barnes et al., 2005). Then the cases were divided into 2 groups: mild dysplasia (MD) and severe dysplasia (SD) groups.

\section{Transmission electron microscopy (TEM)}

The small parts of one cubic mm were fixed in $3 \%$ glutaraldehyde in phosphate buffer $(\mathrm{pH} 7.2)$ for 6 hours. The specimens were then washed in three changes of phosphate buffer. Secondary fixation was achieved in $1 \%$ osmium tetra-oxide at $48{ }^{\circ} \mathrm{C}$ for 1.5 hours and then rinsed in phosphate buffer. Specimens were dehydrated in graded series of ethanol $(50 \%, 70 \%, 85 \%, 95 \%$, and $100 \%)$ for 10 min. at each concentration. The specimens were then cleared in propylene oxide and embedded in epoxy resin. Semi-thin sections of 1-2 mm were cut and stained with $1 \%$ toluidine blue and examined by light microscope for detection of the site to be studied by TEM. Ultra-thin sections were then cut using the ultra microtome (Leica, Germany), mounted on copper grids and stained with uranyl acetate and lead citrate. The grids were examined by Joel 1200 EXII electron microscope (Joel, Peabody, MA, USA). All the procedure was carried out in the National Cancer Institute, Cairo University. 


\section{Immunohistochemistry}

Four microns thick paraffin embedded tissue sections on positively charged glass slides were immunostained with anti-ERK-1 and anti-HIF-1 $\alpha$ antibodies with super sensitive biotin-streptavidin staining technique. Tissue sections were deparafinized, rehydrated, and treated with $0.3 \% \mathrm{H}_{2} \mathrm{O}_{2}$ for $30 \mathrm{~min}$. to block endogenous peroxidase activity. For antigen retrieval, the slides were boiled in $10 \mathrm{mM}$ citrate buffer, $\mathrm{pH} 6.0$ for 10-20min, followed by cooling at room temperature for $20 \mathrm{~min}$.. The positive test slides were incubated with the primary antibodies: mouse monoclonal anti-ERK-1antibody (Cat \# MA1-13041; Thermo Scientific, Labvision, Kalamazoo, MI, USA) and mouse monoclonal antiHIF-1 $\alpha$ antibody (Cat \# MA5-16008; Thermo Scientific, Labvision, Kalamazoo, MI, USA) with the appropriate dilution range 1:100 for $30 \mathrm{~min}$. at room temperature in a humidified chamber. On the other hand, the negative control slides were not exposed to the primary antibodies. After washing with phosphate buffer solution (PBS), the slides were treated with the biotin-labeled link antibody, and then the streptavidin conjugated to horseradish peroxidase was used. The diaminobenzedine (DAB) chromogen was applied to visualize the antigen antibody reaction. All these reagents belong to the universal Labeled Streptavidin-Biotin 2 System, Horseradish Peroxidase (LSAB 2 system-HRP, code \# K0673 DakoCytomation, Glostrup, Denmark). All the slides were immersed in Mayer's hematoxylin for counterstaining. Finally, the sections were covered by cover slips using aqueous mounting medium.

\section{Immunohistochemical evaluation}

The ordinary light microscope was used to detect and localize positive ERK-1 and
HIF-1 $\alpha$ immunostaining. Then, all the sections were examined by an image analyzer computer system using the software Leica Qwin 500 (Leica Microsystems Imaging Solutions Ltd, Cambridge, UK). Five random fields in each specimen were captured using a magnification (x400) to determine the area percentage of the positive cells.

\section{Statistical analysis}

The area percentage of both markers, anti ERK-1 and HIF- $1 \alpha$, in the studied groups, was represented as mean values and standard deviations (SD) for statistical evaluation. The ANOVA test was used to compare the mean values among the control, mild dysplasia and severe dysplasia groups. At 95\% confidence interval, P-value was considered significant when $\mathrm{P}<0.05$.

\section{RESULTS}

\section{I- Electron microscopic findings}

Electron micrographs of $\mathrm{C}$ group showed mitochondria containing a dense matrix and wellorganized inner-membrane cristae, predominantly oriented transverse to the long axis of the mitochondria. The outer and inner mitochondrial membranes appeared intact. Contrarily, mitochondria showed slight changes in the MD group and more obvious changes in SD group in comparison to the $\mathrm{C}$ group. In the former group (MD), they showed slight loss of matrix density, slight degeneration of mitochondrial cristea, as well as being narrower, mis-oriented and reduced in number. On the other hand, SD group showed swollen mitochondria with decreased electron density within the matrix, the cristae were noticeably thinner and often mis-oriented and/or decreased in number (fig. 1). 


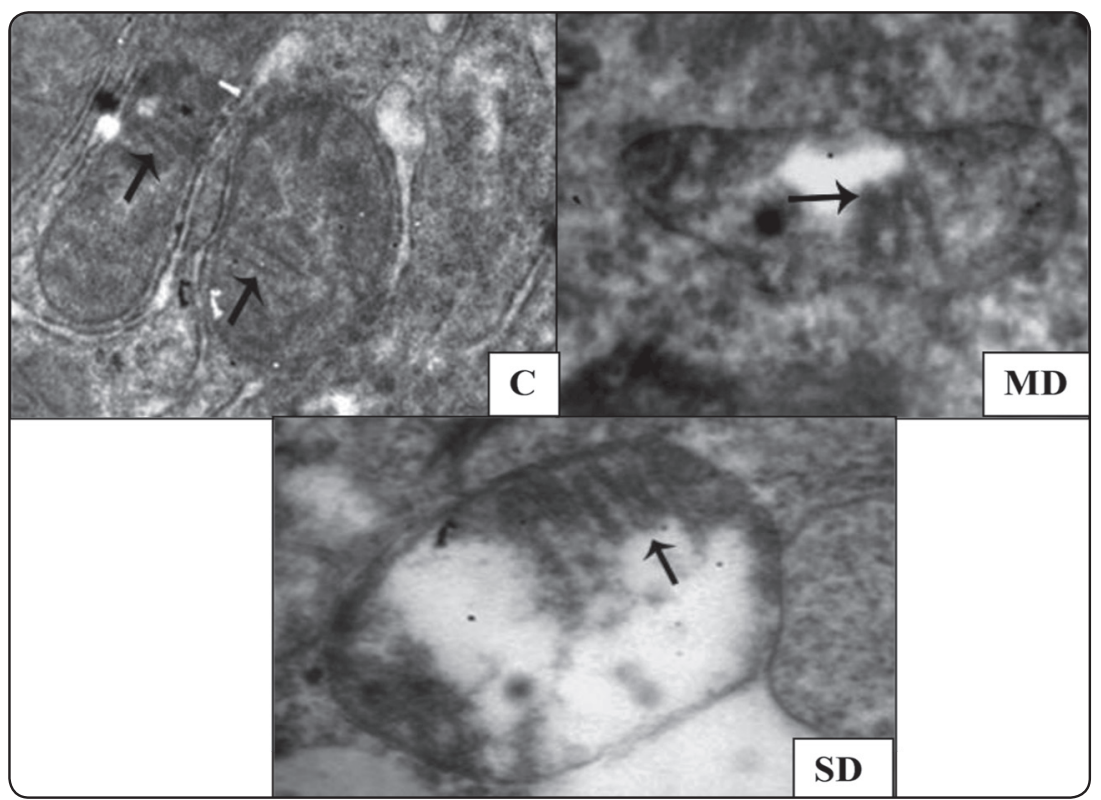

Fig. (1) Electron micrographs showing a dense matrix, well-organized cristae (arrows) and intact outer and inner mitochondrial membranes in ( $\mathrm{C}$ group) (Oig.Mag.X5000), slight loss of matrix density and slightly degenerated, narrower, misoriented and decreased in number mitochondrial cristea (arrow) in (MD group) (Oig.Mag. $\mathrm{X} 10000$ ), swollen mitochondria with marked decrease in matrix density and noticeably thinner, misoriented and decreased number of cristae (arrow) in (SD group) (Oig.Mag.X10000).

\section{II-Histopathological findings}

\section{a- Hematoxylin and eosin stained sections}

The C group (sacrificed at both 6 and 9 week intervals) showed normal keratinized stratified squamous epithelium covering a connective tissue stroma (fig.2a). Concerning the MD group, 100\% of cases sacrificed at 6 weeks interval showed hyperplastic hyperkeratinized stratified squamous epithelium. Twelve out of fifteen cases (80\%) showed clubbing of rete ridges, and some signs of dysplasia (increased N/C ratio, nuclear hyperchromatism and pleomorphism) restricted to the basal $1 / 3$ of the epithelium (fig. 2b). Loss of polarity of the basal cell layer and bizzare mitosis were seen in 4 cases (fig. 2c). The underlying connective tissue (100\% of cases) was infiltrated with few chronic inflammatory cells (figs. 2b\&c).

About $75 \%$ of the cases sacrificed at 9 weeks interval showed hyperplastic stratified squamous epithelium of the tongue with dysplastic features in the form of loss of polarity, cell nest formation, increased N/C ratio, nuclear hyperchromatism and pleomorphism occupying the whole thickness of the epithelium (top to bottom changes) (fig. $3 \mathrm{~d}$ and e). $20 \%$ of the cases $(3 / 15)$ showed loss of continuity of the basement membrane and presence of few epithelial cells in the underlying connective tissue (fig. 3f).

\section{b- ERK-1 immunostained sections}

Positive ERK-1 immunoreactivity was detected in about $75 \%$ of both C and MD groups, and $100 \%$ of SD group. While the ERK-1 positive cells were confined to the basal $1 / 3$ of epithelium in both $\mathrm{C}$ and MD groups, they were observed throughout the epithelium in SD group. In C group, ERK-1 showed faint cytoplasmic and nuclear expression in few cells in the basal 1/3 of the stratified squamous epithelium (fig.3a). The MD group showed weak nuclear immunoexpression in some dysplastic cells of the basal $1 / 3$ of the epithelium (fig. 3b). The SD group showed strong cytoplasmic and nuclear immunoreactivity in dysplastic cells throughout the whole thickness of epithelium (fig. 3c). 


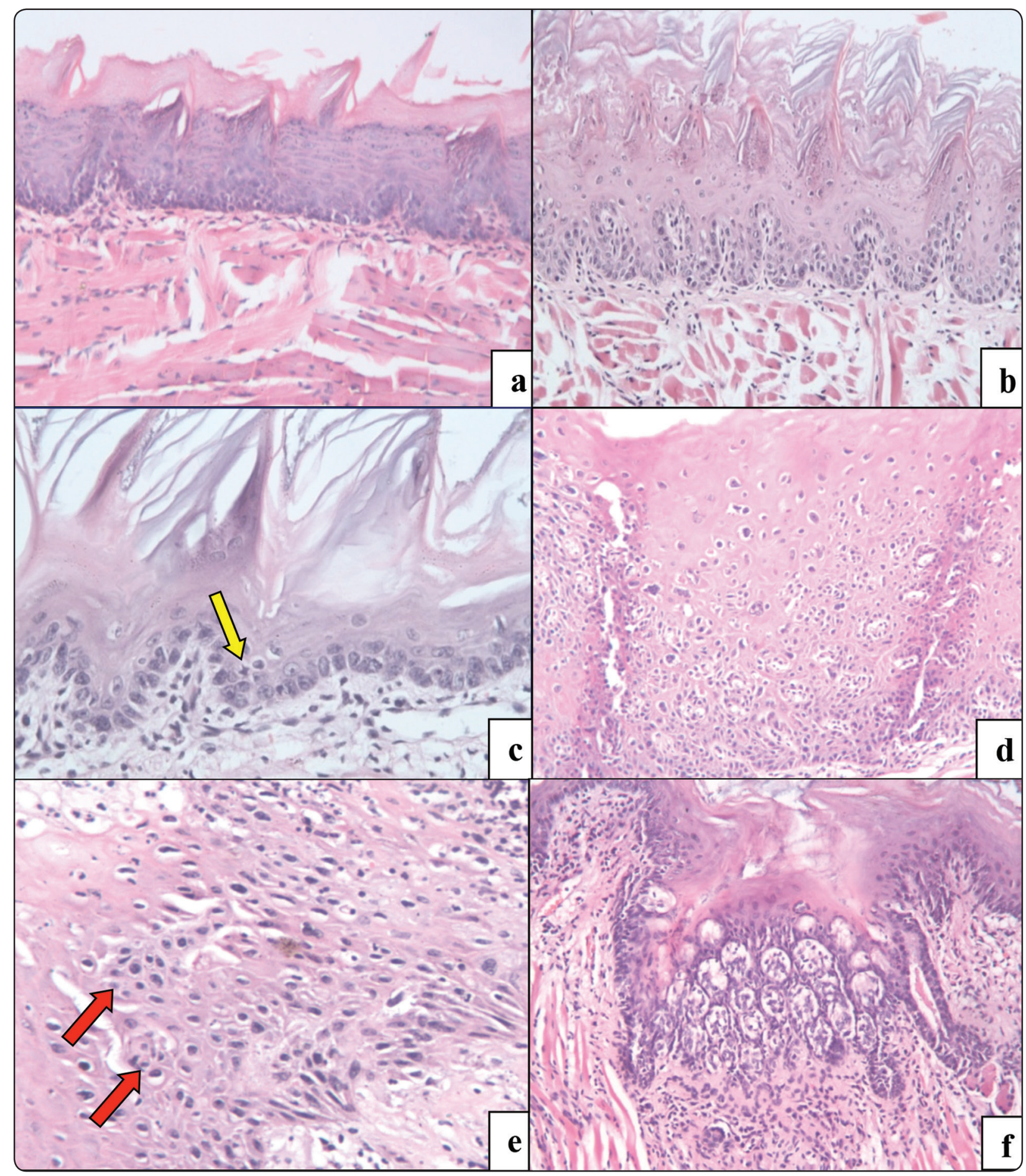

Fig. (2) (a) Normal keratinized stratified squamous epithelium of the dorsal surface of the tongue of C group (H\&Ex200). (b) Hyperplastic hyperkeratinized stratified squamous epithelium with clubbing of the rete ridges and dysplastic features in basal 1/3 of MD group (H\&E x200). (c) Bizarre mitosis is seen (yellow arrow) in basal 1/3 of epithelium of MD group (H\&E x400). (d) Dysplastic features are seen throughout hyperplastic epithelium in SD group (H\&E x200). (e) Cell nest formation (red arrows) in SD group (H\&Ex400). (f) Loss of continuity of the basement membrane and presence of small epithelial masses in the underlying connective tissue are seen (H\&E x200). Note chronic inflammatory cell infiltrate in the underlying connective tissue. 


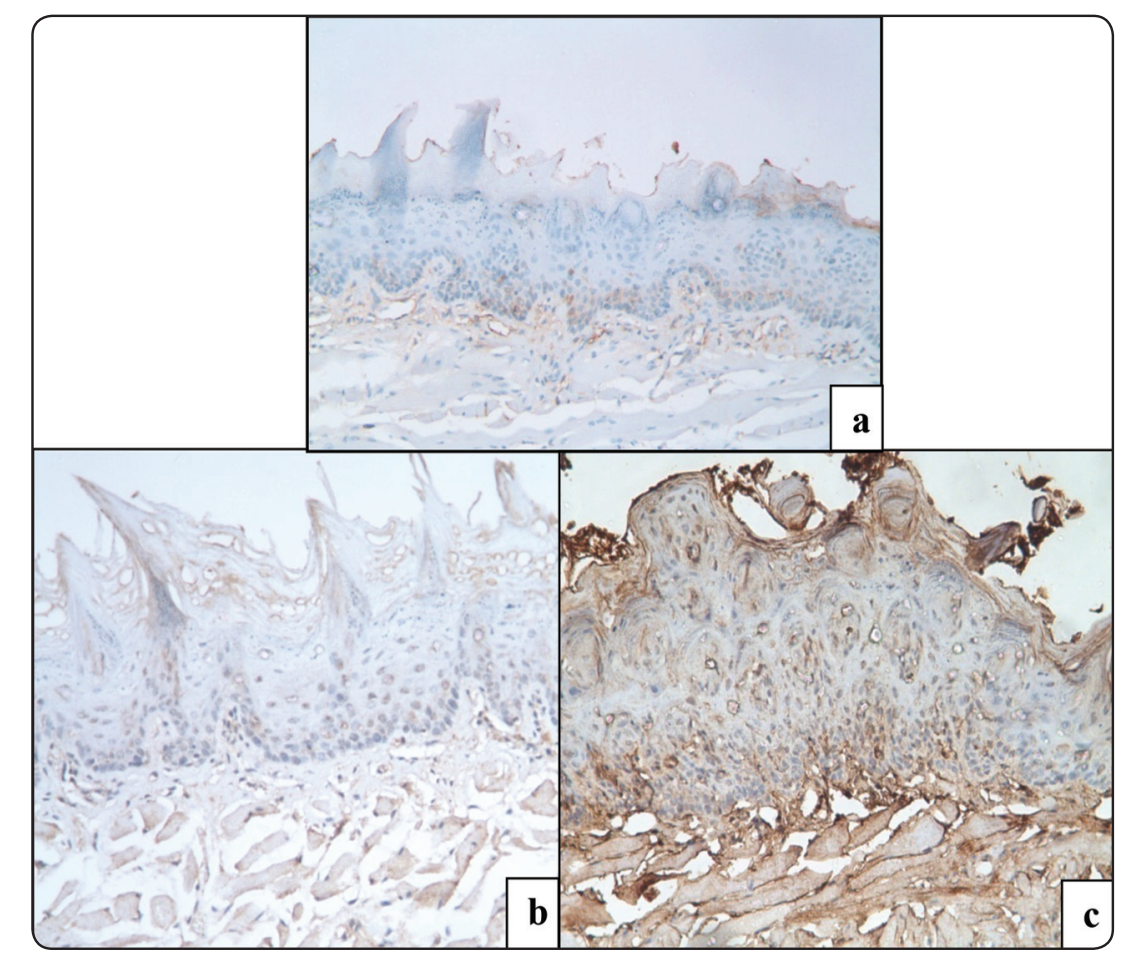

Fig.(3) (a) Faint cytoplasmic and nuclear ERK-1 immunoexpression in the basal 1/3 of stratified squamous epithelium of $\mathrm{C}$ group. (b)Weak nuclear immunoexpression in some dysplastic cells in the basal 1/3 of the epithelium of MD group. (c) In SD group, strong cytoplasmic and nuclear expression in dysplastic cells throughout the whole thickness of epithelium (anti ERK-1 antibody x200).

\section{c- HIF-1 $\alpha$ immunostained sections}

Positive HIF-1 $\alpha$ immunoreactivity was detected in $80 \%$ of $\mathrm{C}$ group, $85 \%$ of MD group, and $100 \%$ of SD group. Unlike ERK-1, the HIF-1 $\alpha$ positive cells were confined to the basal $1 / 3$ of the epithelium in $\mathrm{C}$ group only, and were observed throughout the epithelium in both MD and SD groups (fig. 4). Weak cytoplasmic and nuclear HIF-1 $\alpha$ immunoexpression were observed in few cells in the basal cell layer of stratified squamous epithelium of the $\mathrm{C}$ group (fig. 4a). Strong nuclear and cytoplasmic immunoexpression were detected throughout the epithelium of both MD and SD groups. While the immnuoreaction was mainly nuclear in the MD group (fig. 4b), it was mainly cytoplasmic and membranous in the SD group (figs. $4 \mathrm{c}$ and d).

\section{III-Statistical findings}

A highly significant difference was obtained on comparing the area percentage of positive immunoexpression of both ERK-1 and HIF-1 $\alpha$, where it increased significantly from normal $\mathrm{C}$ group to MD group and reached its highest values in the SD group (Table 1). Although the HIF-1 $\alpha$ showed higher levels in normal $\mathrm{C}$ group and $\mathrm{MD}$ groups, the ERK-1 levels exceeded that of HIF-1 $\alpha$ in the SD group. These values were represented in a bar chart (fig.5). 


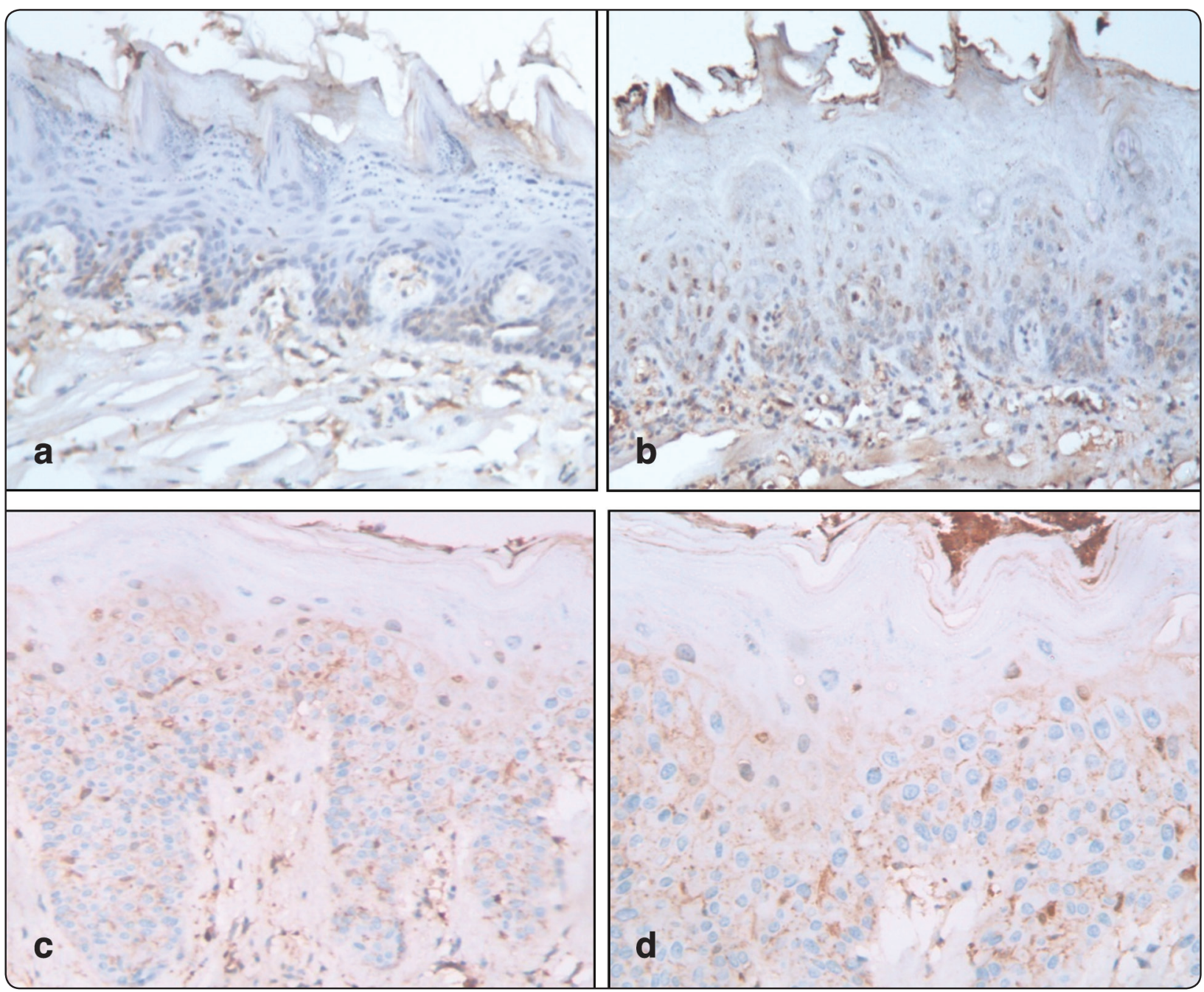

Fig.(4) (a) Weak HIF-1 $\alpha$ immunoexpression in few basal epithelial cells of C group. (b) Strong nuclear and cytoplasmic expression in dysplastic cells throughout the epithelium of MD group. (c) Strong cytoplasmic and membranous expression throughout the epithelium of SD group (anti HIF-1 $\alpha$ x200). (d) Higher magnification of SD group showing nuclear immunoexpression (red arrows) (anti HIF-1 $\alpha \times 400)$.

TABLE (1) Area percentage of positive immunoexpression of both ERK-1 and HIF-1 $\alpha$ among the experimental groups.

\begin{tabular}{|c|c|c|c|}
\hline Groups & C group & MD group & SD group \\
\hline Mean \pm SD of ERK-1 & $1.984 \pm 0.842$ & $9.345 \pm 1.21$ & $37.916 \pm 4.58$ \\
\hline P value & \multicolumn{3}{|c|}{$0.001 * *$} \\
\hline Mean \pm SD of HIF-1 $\alpha$ & $3.822 \pm 1.78$ & $12.34 \pm 1.93$ & $20.484 \pm 5.34$ \\
\hline P value & \multicolumn{3}{|c|}{$0.001 * *$} \\
\hline
\end{tabular}

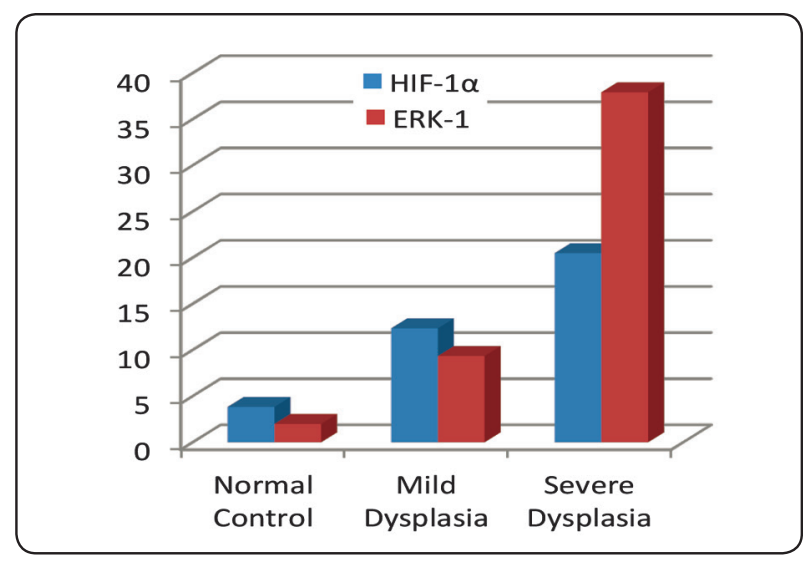

Fig. (5) A bar chart showing the area percentage of positive immunoexpression of both ERK-1 and HIF-1 $\alpha$ among the experimental groups. 


\section{DISCUSSION}

Comprehension of the underlying pathways governing the progression of oral premalignant lesions is of utmost importance. Focus on preinvasive stages of these lesions will allow us to identify early molecular events during cancer development (Banerjee, 2005). The development of oral cancer is a multistep process which includes hyperplasia, dysplasia and finally the tumor. The molecular analysis of these multiple steps is hampered by the unavailability of biopsies of all the stages of carcinogenesis. This highlights the significance of animal models of carcinogenesis that allow the reproducible isolation of all stages, including normal tissues, which are then amenable to pathological, genetic and biochemical analyses (Vairaktaris et al., 2008).

In an attempt to develop oral carcinogenesis in animals, a number of chemical carcinogens have been used as coal tar and cigarette smoke. DMBA is one of the widely used potent carcinogens; it mediates the carcinogenic process by inducing chronic inflammation, over production of ROS and oxidative DNA damage (Suresh et al., 2010).

The mitochondria are the primary target for oxidative damage, leading to alterations in mitochondrial function and morphology (Wang et al., 2012). The structural mitochondrial changes in the present study revealed mitochondrial degeneration, loss of matrix density in addition to narrower, disoriented and reduced number of cristae in association with dysplastic changes which became more obvious in severe dysplasia. These findings are in accordance to Tamgadge et al. (2012) who illustrated that degeneration of mitochondria and disoriented cristae were suggestive of neoplastic changes in oral leukoplakia.

Although mitochondrial morphological changes associated with cancer are varied, the finding of enlarged swollen mitochondria was also reported in pancreatic tumors (Volante et al., 2006) and clear cell carcinoma (Kwon et al., 1996). Furthermore,
Chiche et al. (2010) attributed the unusual enlarged mitochondria to limited oxygen microenvironment. Although inconsistent reports of structural descriptions have rendered TEM valueless in the diagnosis and prognosis of oral precancerous and cancerous lesions (Tamgadge et al., 2012), our work proposed that degenerated mitochondria could be an indicative feature of dysplastic changes.

Concerning ERK-1, up-regulation was detected in the present study, due to increased levels of mitochondrial ROS as adopted by Thannickal and Fanburg (2000). Its overexpression appeared to be related to malignant potential, as reported in many cancerous lesions like OSCC (Mishima et al., 1998), melanoma (Zhuang et al., 2005) and breast cancer (Bartholomeusz et al., 2012). The results of Dong et al. (2014) showed that the increased expression of ERK-1 was closely correlated with malignant transformation of oral leukoplakia. Nuclear and cytoplasmic localization of ERK-1 was detected in the examined groups especially the SD group. This is in accordance to Furukawa et al. (2003) who declared that activation of MAP kinase pathway in the cytoplasm led to ERK-1 activation and its translocation into the nucleus, where it induces various gene expressions leading to cell growth and proliferation.

As regards HIF-1 $\alpha$, overexpression was observed in the current work. This could be attributed to the ability of mitochondria during hypoxia to convey signals to HIF-1 $\alpha$ directly (Solaini et al., 2010) or indirectly through ERK activation which is involved in the hypoxia-induced transcriptional activity of HIF-1 $\alpha$ (Minet et al., 2000 ). Overexpression of HIF-1 $\alpha$ was observed in breast cancer (Costa et al., 2001), OED and OSCC (Zhang et al., 2013). Lin et al. (2008) found that HIF-1 $\alpha$ expression was significantly associated with tumor invasion, lymph node metastasis, clinical stage and prognosis of a variety of human cancers. Similarly, nuclear and cytoplasmic localization of HIF-1 $\alpha$ were also detected. This is in agreement with De Lima 
et al. (2014) who reported HIF-1 $\alpha$ accumulation in the cytoplasm of premalignant cells, then its translocation into the nucleus where it drives the production of stress-adaptive proteins. This response is essential for maintenance of normal oxidative physiology, however it promotes cell survival and proliferation in cancer.

Our results showed a highly significant difference on comparing the area percentage of both ERK-1 and HIF- $1 \alpha$ immunoexpression, where it increased significantly from normal control to mild dysplasia and reached its highest values in the severe dysplasia group. This is in accordance with Lin et al. (2008) who illustrated a significant increase in HIF-1 $\alpha$ labeling indices in normal oral epithelium through oral epithelial dysplasia and OSCC. Although the HIF- $1 \alpha$ showed higher levels in normal control and mild dysplasia groups, the ERK-1 levels exceeded that of HIF- $1 \alpha$ in the severe dysplasia group. This could shed the light on the importance of ERK1 as a more potent early predictor of malignant transformation.

Taken together, our findings have shown that altered mitochondrial ultrastructure as well as overexpression of hypoxia related proteins (ERK-1 and HIF-1 $\alpha$ ) could be indicative features of dysplastic changes, might play important roles in progression of oral carcinogenesis and act as valuable prognostic markers. Their overexpression represented an early event in oral carcinogenesis that could be employed as new therapeutic strategies in the future.

\section{REFERENCES}

- Banerjee AG, Bhattacharyya I, Vishwanatha JK: Identification of Genes and Molecular Pathways Involved in the Progression of Premalignant Oral epithelia. Molecular Cancer Therapy, 4(6):865-875, 2005.

- Barnes L, Eveson JW, Reichart P, Sidransky D: World Health Organization Classification of Tumors. Pathology and Genetics of Head and Neck Tumors. IARC Press, Lyon, 209-281, 2005.

- Bartholomeusz C, Gonzalez-Angulo AM, Liu P, et al.: High ERK Protein Expression Levels Correlate with
Shorter Survival in Triple-Negative Breast Cancer Patients. Oncologist, 17(6):766-774, 2012.

- Chiche J, Rouleau M, Gounon P, Brahimi-Horn MC, Pouysségur J, Mazure NM: Hypoxic Enlarged Mitochondria Protect Cancer Cells from Apoptotic Stimuli. Journal of Cell Physiology, 222(3):648-657, 2010.

- Costa A, Coradini D, Carrassi A, Erdas R, Sardella A , Daidone MG: Levels of Hypoxia-Inducible Factor- $1 \alpha$ During Breast Carcinogenesis. Journal of the National Cancer Institute, 93(15):1175-1177, 2001

- De Lima PO, Jorge CC, Oliveira DT, Pereira MC: Hypoxic Condition and Prognosis in Oral Squamous Cell Carcinoma. Anticancer Research, 34: 605-612, 2014.

- Dimova EY, Michiels C, Kietzmann T: Kinases as Upstream Regulators of the HIF System: Their Emerging Potential as Anti-Cancer Drug Targets. Current Pharmaceutical Design, 15(33):3867-3877, 2009.

- Dong Y, Li Z, Gao M, et al.: Immunohistochemical Detection of Aurora A and ERK Pathway in Oral Leukoplakia and Oral Squamous Cell Carcinoma. Journal of Hard Tissue Biology, 23(1):71-76, 2014.

- $\quad$ Furukawa T, Sunamura M, Motoi F, Matsuno S, Horii A: Potential Tumor Suppressive Pathway Involving DUSP6/ MKP-3 in Pancreatic Cancer. American Journal of Pathology, 162(6): 1807-1815, 2003.

- Hileman EO, Liu J, Albitar M, Keating MJ, Huang P: Intrinsic Oxidative Stress in Cancer Cells: A Biochemical Basis for Therapeutic Selectivity. Cancer Chemotherapy and Pharmacology, 53 (3): 209-219, 2004.

- Kwon TJ, Ro JY, Mackay B: Clear Cell Carcinoma: An Ultrastructural Study of 57 Tumors from various sites. Ultrastructural Pathology, 20(6):519-527, 1996.

- $\quad$ Lin PY, Yu CH, Wang JT, et al.: Expression of HypoxiaInducible Factor-1 Alpha is Significantly Associated with the Progression and Prognosis of Oral Squamous Cell Carcinomas in Taiwan. Journal of Oral Patholology and Medicine, 37(1):18-25, 2008.

- Meloche S, Pouysségur J: The ERK1/2 Mitogen-Activated Protein Kinase Pathway as a Master Regulator of the G1To S-Phase Transition. Oncogene, 26: 3227-3239, 2007.

- Minet E, Arnould T, Michel G, et al.: ERK Activation upon Hypoxia: Involvement in HIF-1 Activation. FEBS Letters, 468: 53-58, 2000.

- Mishima K, Yamada E, Masui K, et al.: Overexpression of the ERK/MAP Kinases in Oral Squamous Cell Carcinoma. Modern Pathology, 11(9):886-891, 1998. 
- Pavithra S: Antioxidants in Oral Healthcare. Journal of Pharmaceutical Sciences \& Research, 6(4): 206-209, 2014.

- Reuter S, Gupta SC, Aggarwal BB: Oxidative stress, inflammation, and cancer: How are they linked? Free Radical Biology \& Medicine, 49(11):1603-1616, 2010.

- Solaini G, Baracca A, Lenaz G, Sgarbi G: Hypoxia and Mitochondrial Oxidative Metabolism. Biochimica et Biophysica Acta (BBA) - Bioenergetics, 1797(6-7): 1171-1177, 2010.

- $\quad$ Suresh K, Sivakumar K, Vijayaanand MA, Rajalingam K, Rajkamal G: Anti-Lipid Peroxidative and Antioxidant Effects of Zingiber Officinale Roscoe Root Extract in 7, 12-Dimethyl Benz[A] Anthracene Induced Oral Carcinogenesis. Pharmacology Online, 2: 689-701, 2010.

- Tamgadge SA, Ganvir SM, Hazarey VK, Tamgadge A: Oral Leukoplakia: Transmission Electron Microscopic Correlation With Clinical Types and Light Microscopy. Dental Research Journal (Isfahan), 9(1): S94-S104, 2012.

- Thannickal VJ, Fanburg BL: Reactive oxygen species in cell signaling. American Journal of Physiology, 279: (6): L1005-L1028, 2000.

- Tilakaratne WM, Nissanka-Jayasuriya EH: Value of HIF- $1 \alpha$ as an Independent Prognostic Indicator In Oral Squamous Cell Carcinoma. Expert Review of Molecular Diagnostics, 11(2):145-147, 2011.

- Yu M: Generation, Function and Diagnostic Value of Mitochondrial DNA Copy Number Alterations in Human Cancers. Life Science, 89: 65-71, 2011.
- Vairaktaris E, Spyridonidou S, Papakosta V, et al.: The Hamster Model of Sequential Oral Oncogenesis. Oral Oncology, 44(4):315-324, 2008.

- Volante M, La Rosa S, Castellano I, Finzi G, Capella C, Bussolati G: Clinico-Pathological Features of a Series of 11 Oncocytic Endocrine Tumours of the Pancreas. Virchows Archive, 448(5):545-551, 2006.

- Wang GC, Hazra TK, Mitra S, Lee HM, Englander EW: Mitochondrial DNA Damage and a Hypoxic Response are Induced by Coci2 in Rat Neuronal PC12 Cells. Nucleic Acids Research, 28: 2135-2140, 2000.

- Wang Y, Nartiss Y, Steipe B, McQuibban GA, Kim PK: ROS-Induced Mitochondrial Depolarization Initiates PARK2/PARKIN-Dependent Mitochondrial Degradation by Autophagy. Autophagy, 8(10), 1462-1476, 2012.

- Zhang X, Han S, Han HY, et al.: Risk Prediction for Malignant Conversion of Oral Epithelial Dysplasia by Hypoxia Related Protein Expression. Pathology, 45(5):478-483, 2013.

- Zhuang L, Lee CS, Scolyer RA, et al.: Activation of the Extracellular Signal Regulated Kinase (ERK) Pathway in Human Melanoma. Journal of Clinical Pathology, 58(11): 1163-1169, 2005.

- Ziech D, Franco R, Pappa A, Panayiotidis MI: Reactive Oxygen Species (ROS)--Induced genetic and epigenetic alterations in human carcinogenesis. Mutation Research/ Fundamental and Molecular Mechanisms of Mutagenesis, 711(1-2, 3):167-173, 2011. 